\title{
The Role of Hydration in Protein Stability: Comparison of the Cold and Heat Unfolded States of Yfh1
}

\section{Miquel Adrover ${ }^{1,2}$, Gabriel Martorell ${ }^{3}$, Stephen R. Martin ${ }^{1}$, Dunja Urosev ${ }^{4}$, Petr V. Konarev ${ }^{5}$, Dmitri I. Svergun ${ }^{5}$, Xavier Daura ${ }^{4,6}$, Pierandrea Temussi ${ }^{1,7}$ and Annalisa Pastore ${ }^{1 *}$}

\author{
${ }^{1}$ National Institute for Medical Research, The Ridgeway, London NW7 1AA, UK \\ ${ }^{2}$ Department de Química, Universitat de les Illes Balears, Palma de Mallorca E-07122, Spain \\ ${ }^{3}$ Serveis Científico-Tècnics, Universitat de les Illes Balears, Palma de Mallorca E-07122, Spain \\ ${ }^{4}$ Institute of Biotechnology and Biomedicine, Universitat Autònoma de Barcelona, Bellaterra E-08193, Spain \\ ${ }^{5}$ European Molecular Biology Laboratory, EMBL c/o DESY, Notkestrasse 85, Hamburg D-22603, Germany \\ ${ }^{6}$ Catalan Institution for Research and Advanced Studies (ICREA), Barcelona E-08010, Spain \\ ${ }^{7}$ Dipartimento di Chimica, Universita' di Napoli Federico II, Via Cinthia, I-80126 Napoli, Italy
}

Received 5 December 2011; accepted 1 February 2012

Available online

14 February 2012

\section{Edited by B. Honig}

Keywords:

cold denaturation;

frataxin;

NMR;

protein stability;

SAXS
Protein unfolding occurs at both low and high temperatures, although in most cases, only the high-temperature transition can be experimentally studied. A pressing question is how much the low- and high-temperature denatured states, although thermodynamically equivalent, are structurally and kinetically similar. We have combined experimental and computational approaches to compare the high- and low-temperature unfolded states of Yfh1, a natural protein that, at physiologic $\mathrm{pH}$, undergoes cold and heat denaturation around $0{ }^{\circ} \mathrm{C}$ and $40{ }^{\circ} \mathrm{C}$ without the help of ad hoc destabilization. We observe that the two denatured states have similar but not identical residual secondary structures, different kinetics and compactness and a remarkably different degree of hydration. We use molecular dynamics simulations to rationalize the role of solvation and its effect on protein stability.

Crown Copyright (c) 2012 Published by Elsevier Ltd. All rights reserved.

\section{Introduction}

Several decades of biophysical studies have led to the identification of the main forces that govern protein stability. Yet, many phenomena remain poorly understood, particularly those connected with the action of intramolecular and intermolecular interactions at low temperature. The temperature

*Corresponding author. Annalisa Pastore, National Institute for Medical Research, The Ridgeway, London NW7 1AA, UK. E-mail address: apastor@nimr.mrc.ac.uk.

Abbreviations used: SAXS, small-angle X-ray scattering; MD, molecular dynamics; HSQC, heteronuclear single quantum coherence; SSP, secondary structure propensity; EOM, ensemble optimization method. range of protein stability in the native folded state, largely investigated at temperatures above room temperature, can vary significantly for different proteins. It is, however, widely recognized that proteins undergo also a "cold denaturation," that is, a transition from a folded to an unfolded state at temperatures below room temperature. ${ }^{1}$

Understanding whether the two transitions, which are thermodynamically equivalent, are also similar from the structural and kinetic point of view is a fascinating topic, which is still actively debated. It has been suggested, for instance, that while heat denaturation is generally highly cooperative, cold denaturation might occur in a noncooperative fashion. ${ }^{2}$ An additional burning question is the role played by the solvent in the two transitions. The currently accepted hypothesis is that the main 
driving force for cold denaturation is the favorable enthalpic contribution of hydration of apolar groups at low temperature. ${ }^{1}$

A definitive experimental proof of these theories is still missing: cold denaturation is difficult to study since, for most proteins, it occurs at temperatures much lower than $0{ }^{\circ} \mathrm{C}$, that is, under conditions in which aqueous solutions are frozen. An elegant but cumbersome way to circumvent this difficulty is to keep water under a supercooled condition. ${ }^{3}$ Alternatively, the temperature of cold denaturation can be raised by destabilizing the protein, for instance, by mutations or solvent perturbation. The main limitation of studying artificially denatured proteins is, however, the difficulty of extrapolating the results to physiological conditions. A noninvasive and facile way to observe cold denaturation was found with the identification of a protein, Yfh1, whose cold denaturation occurs at neutral $\mathrm{pH}$ and without addition of destabilizing agents at temperature sufficiently high to be easily attainable experimentally. ${ }^{4}$ Yfh 1 is the yeast ortholog of frataxin, a mitochondrial human protein involved in Friedreich's ataxia, a severe neurodegenerative disease. ${ }^{5}$ The frataxin family is highly conserved in both sequence and structure from bacteria to humans and is essential for life. Despite the high conservation, the thermodynamic stability of different frataxin orthologs varies appreciably and is strongly influenced by iron and other cations. ${ }^{6}$ Yfh 1 is the member of the frataxin family so far explored with the highest and lowest melting points at low and high temperatures, respectively, around 7 and $30^{\circ} \mathrm{C}$ when in its salt-free form. Its thermal stability, together with the fact that it is a protein from natural sources rather than an "ad hoc" designed mutant, makes Yfh1 a system uniquely suited for an extensive characterization of the cold transition and its related phenomena. Using this system, we have, for instance, been able to show that alcohols, rightly considered as denaturing agents at high concentrations, behave as stabilizers of native protein folds at low concentration and low temperature. $^{7}$

The unbiased cold denaturation of Yfh1 is thus also ideally suited for addressing the question of whether the unfolded state is structurally the same at high and low temperatures. We have recently reported a nuclear magnetic resonance (NMR) study of the cold denatured state. ${ }^{8}$ Here, we compare the structural features of the cold and heat denaturated states of Yfh1 using a combination of circular dichroism (CD), NMR and small-angle X-ray scattering (SAXS) techniques and analyze the experimental findings by molecular dynamics (MD) simulations. We conclude that the two states are mostly structurally equivalent but have a different SAXS profile, indicating very different population distributions, and experience a different degree of hydration, which is higher at low temperature. MD simulations have helped us to rationalize our experimental findings by showing quantitatively a longer persistence of the hydrogen bonding with the solvent at low temperature. Our data provide the first experimental support to the hypothesis that cold denaturation is caused by a hydration increase.

\section{Results}

\section{Yfh1 unfolding occurs in a slow equilibrium exchange regime and is not symmetric}

To compare the low- and high-temperature denaturing processes, we first followed the whole thermal stability curve by CD spectroscopy and compared the data obtained by heating and successively cooling the sample (Fig. 1a). The process is highly reversible, thus excluding any interference of aggregation. The thermal unfolding process recorded on different protein batches is also highly reproducible and can reliably be interpreted as a two-state transition with transition points around 7 and $30{ }^{\circ} \mathrm{C}$ and the point of maximal stability around $19{ }^{\circ} \mathrm{C} .{ }^{4}$ We estimated elsewhere that, at the point of maximal stability, ca $20 \div 30 \%$ of the protein is unfolded in rough agreement with the presence of $15 \div 25$ (depending on the structure used as reference ${ }^{9,10}$ ) unstructured residues that belong to the N-terminal tail that contains the processed part of the mitochondrial import signal and the presence of unstructured loops. ${ }^{11}$

We first tried to select equivalent states devoid of residual folded populations at low and high temperatures by back calculations based on the published thermodynamic parameters. ${ }^{4,7} \mathrm{CD}$ suggests $-5{ }^{\circ} \mathrm{C}$ and $45^{\circ} \mathrm{C}$ as equivalent points along the unfolding curve, being roughly equidistant from the point of maximal stability and from the midpoints of unfolding. At these temperatures, the residual population of folded Yfh1 is estimated to be ca $7 \%$, which should be hardly detectable by most of the standard commercial NMR instruments.

However, the ${ }^{1} \mathrm{H}^{15} \mathrm{~N}$ heteronuclear single quantum coherence (HSQC) NMR spectra recorded at different temperatures tell us a different story (Fig. 1b). Unfolding occurs at both low and high temperatures by a slow exchange regime with the folded species progressively disappearing while the unfolded ones appear. The HSQC spectrum of Yfh1 at $-1{ }^{\circ} \mathrm{C}$, corresponding to a theoretical residual folded population of $19 \%$, is instead already typical of fully denatured species with little resonance dispersion. The spectrum at $45{ }^{\circ} \mathrm{C}$ contains two populations: a dominant one that accounts for the low dispersion resonances in co-presence with a residual population of folded species at positions 
(a)

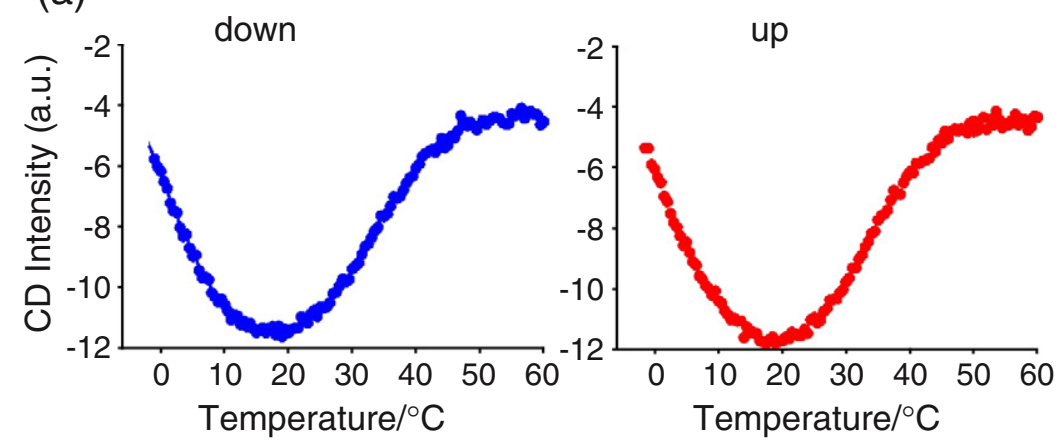

(b)

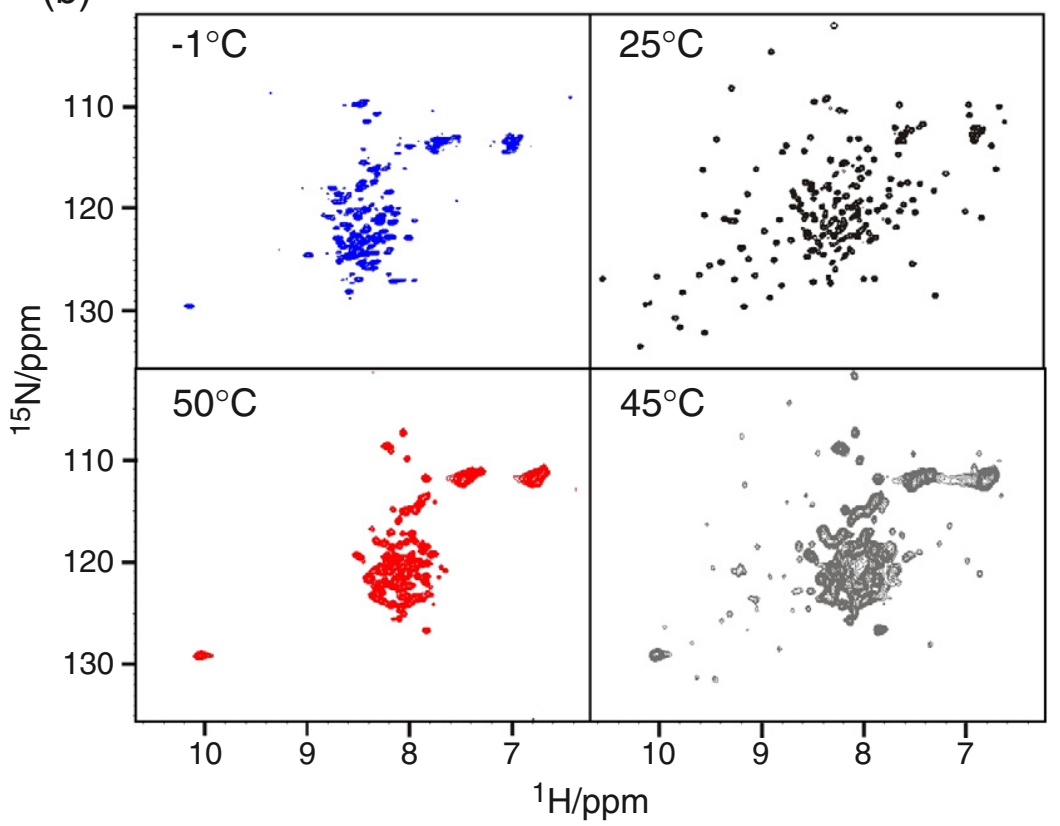

Fig. 1. Choosing equivalent unfolded states. (a) Plot of the CD signal intensity (a.u.) of Yfh1 at 222 $\mathrm{nm}$ as a function of temperature. The blue curve (left) was recorded by decreasing the temperature, and the red curve (right) was obtained by raising the temperature. (b) ${ }^{15} \mathrm{~N}$ HSQC spectra of Yfh1 at four different temperatures: $-1{ }^{\circ} \mathrm{C}$, $25^{\circ} \mathrm{C}, 45^{\circ} \mathrm{C}$ and $50^{\circ} \mathrm{C}$, respectively, clockwise.

very similar to those observed at $25{ }^{\circ} \mathrm{C}$. It is only around $50^{\circ} \mathrm{C}$ that the spectrum collapses completely and the folded population is no longer detectable.

These data strongly suggest that the two states experience a different kinetic of folding/unfolding interconversion, with the low-temperature state being immediately trapped in the unfolded state, while the high temperature can revert into the folded state. All the following comparative studies were carried out on "pure" unfolded states, that is, at $-1{ }^{\circ} \mathrm{C}$ and $50{ }^{\circ} \mathrm{C}$.

\section{The two denatured states differ in their residual structure}

The low dispersion and the absence of ring current highfield shifted peaks in the spectra at -1 and $50^{\circ} \mathrm{C}$. It is, however, well known that there is often residual secondary structure even in intrinsically unfolded proteins. ${ }^{12}$ To further characterize the two denatured states, we repeated the spectral assignment at high temperature using the same strategy previously described for the assignment of the spectrum at low temperature. ${ }^{8}$ We were able to achieve complete assignment at $50{ }^{\circ} \mathrm{C}$ for $91 \%$ of the residues and partial assignment (i.e., $\mathrm{HA}, \mathrm{HB}, \mathrm{CA}$ and $\mathrm{CB}$ resonances) for $7 \%$ of the remaining residues, leaving only a bare $2 \%$ unassigned (Fig. 2a). Thanks to a more efficient magnetization transfer, some regions (i.e., residues 70-80 and 90-93) could not be assigned at low temperature but were reliably identified at high temperature.

We tried to detect residual secondary structure by comparing the nuclear Overhauser enhancement patterns observed in nuclear Overhauser enhancement spectroscopy-HSQC spectra. ${ }^{13}$ However, spectra recorded at low and high temperatures are very different: the spectrum at $-1{ }^{\circ} \mathrm{C}$ is well populated and informative, even though most of the nuclear Overhauser enhancement effects are intra-residue or sequential as previously reported. ${ }^{8}$ The $50{ }^{\circ} \mathrm{C}$ nuclear Overhauser enhancement spectroscopy-HSQC spectrum contains instead very few off-diagonal peaks (Fig. S1 of SI). This is consistent 
(a)

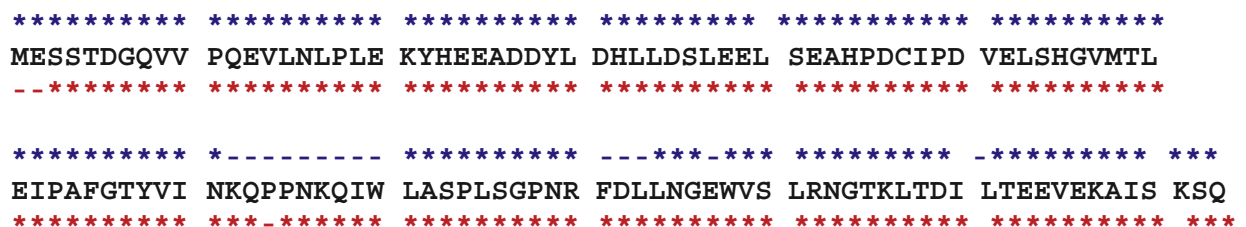

(b)

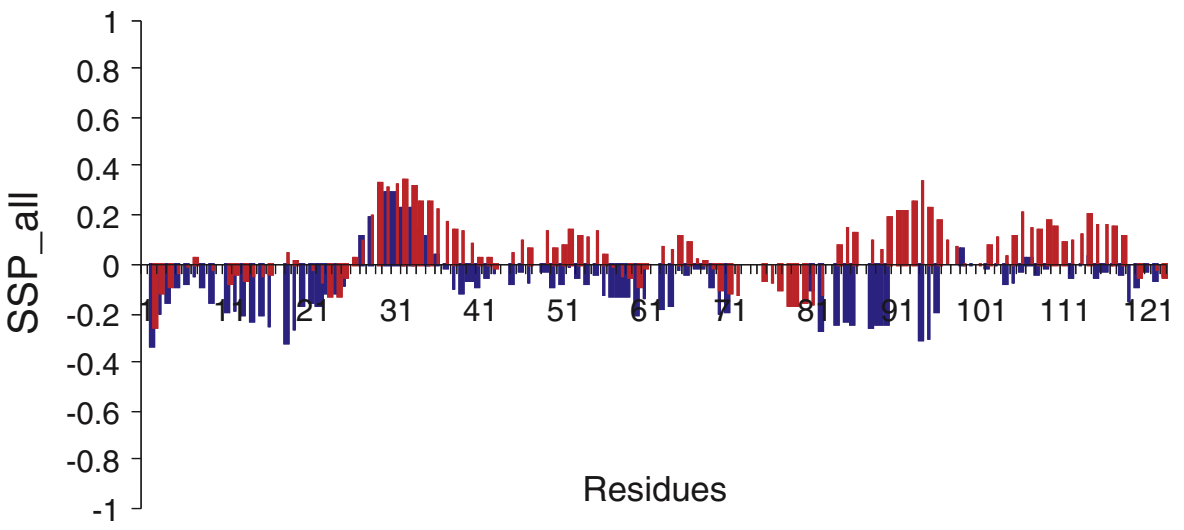

Fig. 2. Characterization of the two denatured states by NMR. (a) Yfh1 primary sequence on which we have indicated the residues for which NMR spectral assignment was achieved. Blue and red asterisks refer to the assignment at $-1{ }^{\circ} \mathrm{C}$ and at $50^{\circ} \mathrm{C}$, respectively. Missing assignments are indicated by a dash. (b) SSP plot calculated for the HN, N, CA, HA and $\mathrm{CB}$ groups at $-1{ }^{\circ} \mathrm{C}$ (blue histogram) and $50{ }^{\circ} \mathrm{C}$ (red histogram).

with a different exchange regime, probably with the solvent, at the two temperatures.

We then explored the information embedded in the chemical shifts. ${ }^{14}$ It is customary to identify secondary structural elements by calculating a chemical shift index. ${ }^{15}$ However, it has been correctly pointed out that, in unfolded proteins, the use of the chemical shift index could be inappropriate because it might be too rough a parameter to reflect only transiently formed residual secondary structure. ${ }^{14}$ It is preferable to evaluate elements of secondary structure by secondary chemical shifts ${ }^{16}$ that represent the deviation of the chemical shifts at a given temperature from random-coil chemical shift values at $25^{\circ} \mathrm{C}$. The most sensitive way to exploit this information uses a combination of them as in the calculation of the secondary structure propensity (SSP) score that makes the results more robust against spectrum referencing problems. ${ }^{17}$ The SSP plot and secondary chemical shifts along the sequence calculated assuming random-coil values obtained from model peptides ${ }^{14,18}$ at $-1^{\circ} \mathrm{C}$ and at $50{ }^{\circ} \mathrm{C}$ are qualitatively similar, although with interesting local differences (Fig. 2b). The overall behavior suggests that the N-terminal tail adopts local structure at low temperature but not at $50^{\circ} \mathrm{C}$ where it has SSP values close to the random coil. At both temperatures, helix 1 (residues 19-43) has higher persistence than the C-terminal helix (residues 107-119), which almost completely unfolds at low temperature. The five-strand $\beta$-sheet mostly unfolds except around residues 80-100 where it adopts different residual transient structure at the two temperatures.

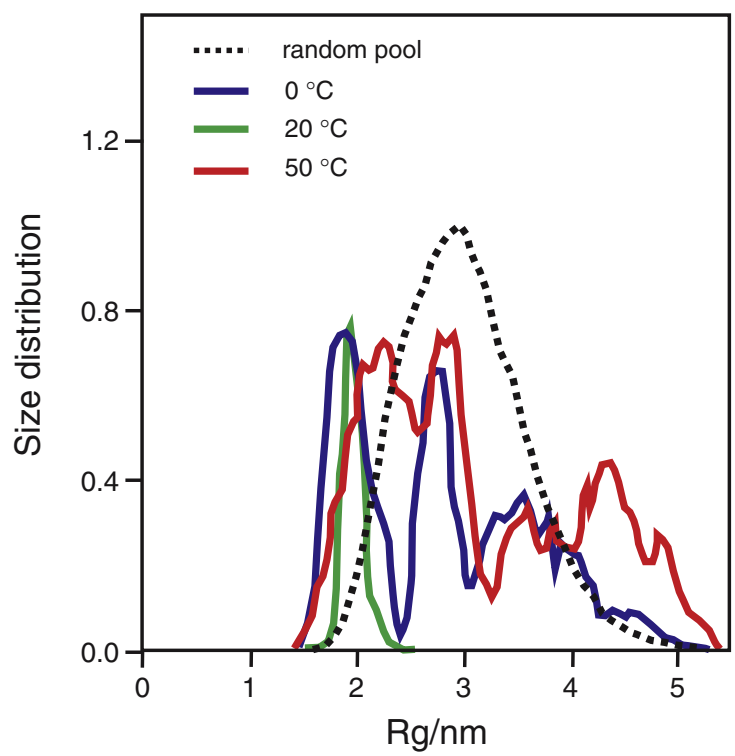

Fig. 3. Size distributions as obtained by SAXS. The plots were calculated by the EOM analysis of the Yfh1 data recorded at -1 (blue), 20 (dark green) and $50^{\circ} \mathrm{C}$ (red). They are compared with the distribution calculated by assuming that Yfh1 is all in a pure disordered state (black broken line). 
(a)

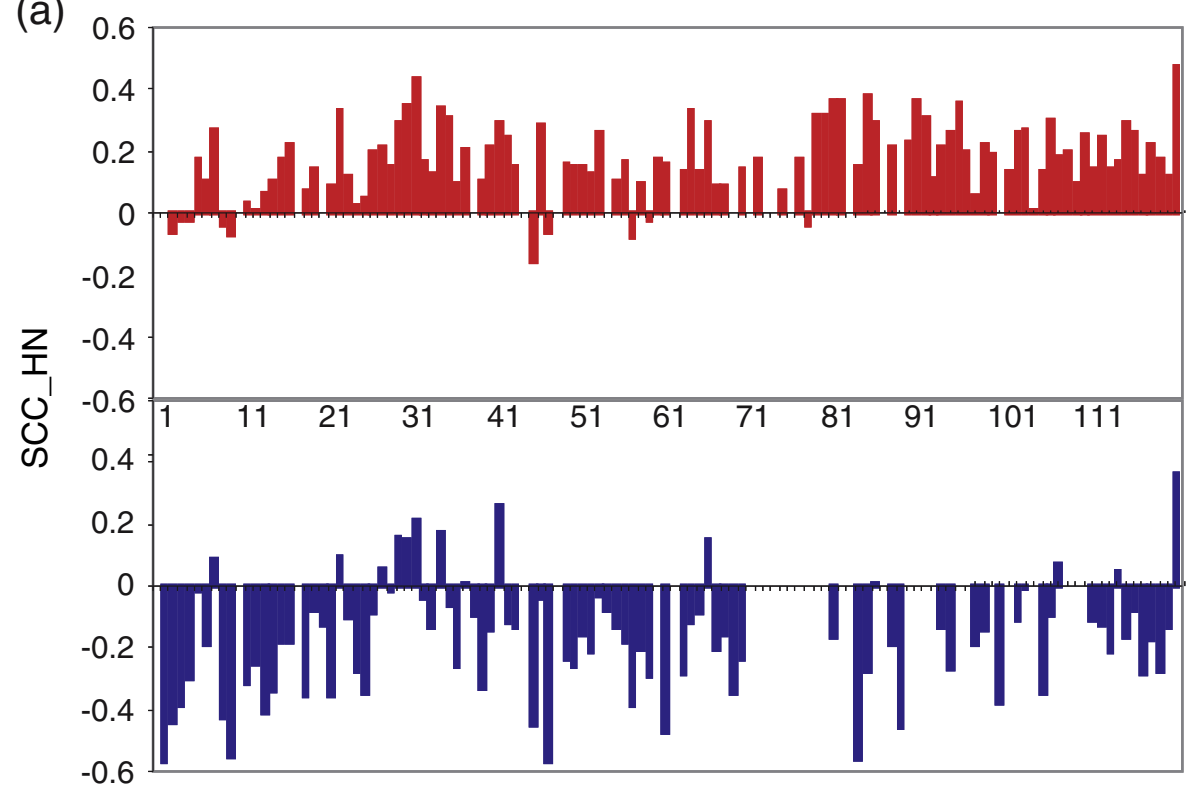

(b)

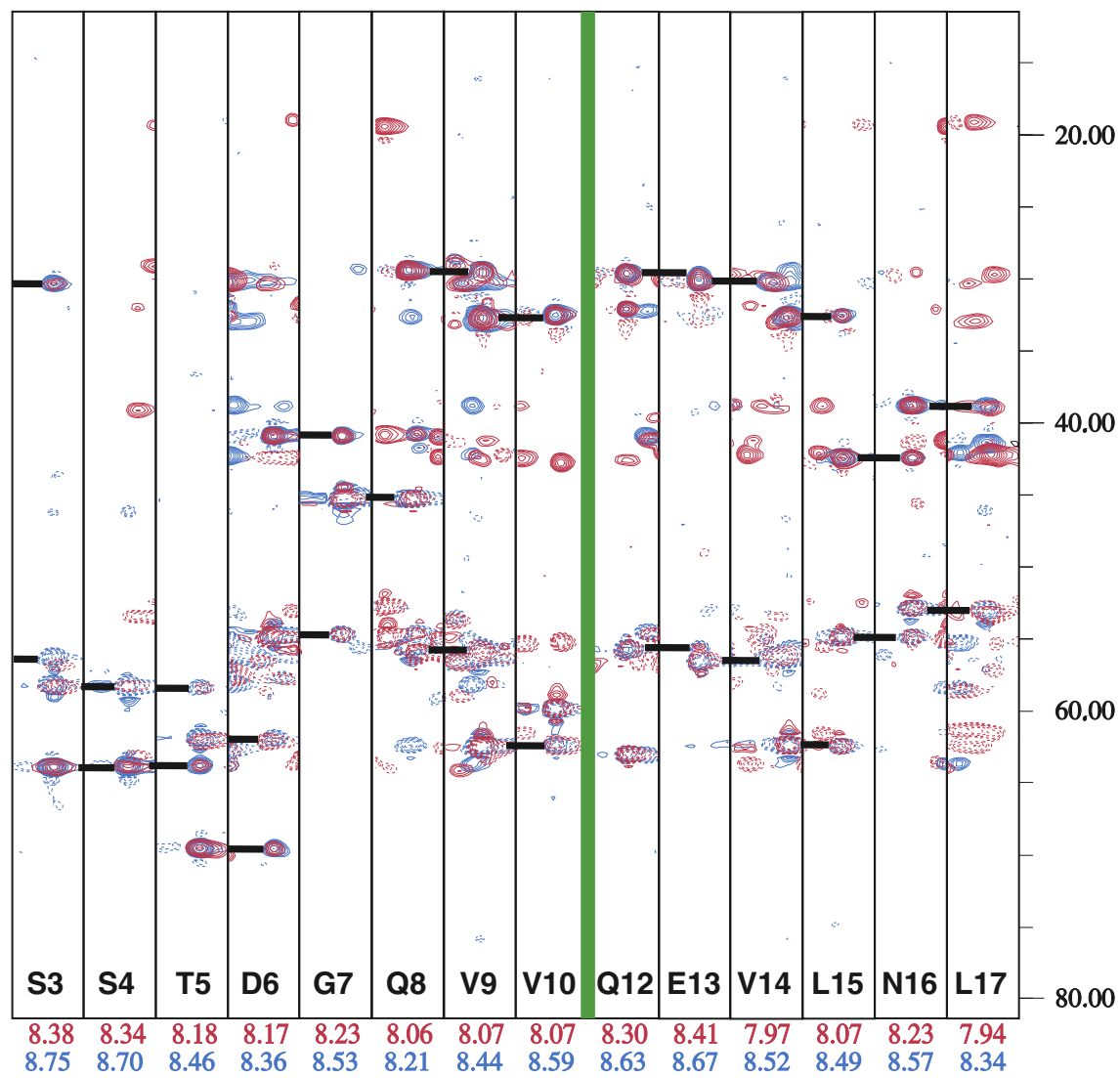

Fig. 4. Comparison of the HN chemical shifts of Yfh1. (a) Secondary chemical shifts $\left(\delta_{25}{ }^{\circ} \mathrm{C}-\delta_{\mathrm{T}}\right)$ at $-1{ }^{\circ} \mathrm{C}$ (blue histogram) and $50{ }^{\circ} \mathrm{C}$ (red histogram). The sign is consistently reversed in the two plots. (b) Superposition of representative strips from HNCACB spectra at two temperatures. Strips at $-1{ }^{\circ} \mathrm{C}$ are colored blue, whereas the corresponding ones at $50{ }^{\circ} \mathrm{C}$ are colored red. Although carbon peaks superimpose well, the corresponding $\mathrm{HN}$ values (reported at the basis of the strips in red and blue for $50^{\circ} \mathrm{C}$ and $-1{ }^{\circ} \mathrm{C}$, respectively) differ on average by $0.2-0.4$. The green vertical bar marks the position of P11. 
These results tell us that the two denatured states have individual structural features.

\section{The two denatured states have different gyration radii and equilibrium states}

To further characterize the two unfolded species, we used SAXS, a technique that can quantitatively describe overall structure parameters (e.g., the radius of gyration of a protein, $R_{\mathrm{g}}$ ) and the structural transitions from native states to partially or completely unfolded proteins. ${ }^{19}$ The $\mathrm{Yfh} 1$ scattering patterns were measured at $0,10,20,40$ and $50{ }^{\circ} \mathrm{C}$ (Fig. S2 of SI, left panel). The corresponding $R_{\mathrm{g}}$ values are $2.20 \pm 0.05 \mathrm{~nm}$ at $0{ }^{\circ} \mathrm{C}, 2.10 \pm 0.05 \mathrm{~nm}$ at $20^{\circ} \mathrm{C}$ and $2.50 \pm 0.05 \mathrm{~nm}$ at $50{ }^{\circ} \mathrm{C}$. The data at 10 and $40{ }^{\circ} \mathrm{C}$ had features very close to those at $20^{\circ} \mathrm{C}$, supporting the very sudden collapse of the fold. The Kratky plots (Fig. S2 of SI, right panel) are consistent with unfolded but collapsed states at 0 and $50{ }^{\circ} \mathrm{C}$.

The ensemble optimization method $(\mathrm{EOM})^{20}$ was applied to quantitatively characterize the ensemble properties of these states under equilibrium conditions (Fig. 3). The average sizes and the $R_{\mathrm{g}}$ distributions of the population ensembles are clearly affected by temperature. The $R_{\mathrm{g}}$ distribution at $20{ }^{\circ} \mathrm{C}$ is consistent with a well-defined compact folded conformation, as also judged by the Kratky plots (Fig. S2 of SI, right panel). Best fit of the data at this temperature is obtained by assuming the first 30 residues unfolded and flexible, in agreement with the estimate from the $\Delta G$ curve. ${ }^{4}$ The ensemble distributions selected by the EOM analysis at 0 and $50{ }^{\circ} \mathrm{C}$ are shifted to larger $R_{\mathrm{g}}$ values but with important differences. The $R_{\mathrm{g}}$ distribution at $50^{\circ} \mathrm{C}$ is closer to the one of a completely random pool, whereas that at $0{ }^{\circ} \mathrm{C}$ has a significant fraction of compact and folded conformations (corresponding to the peak around $2 \mathrm{~nm}$ ).

These data indicate that, despite the local structural similarity, the two cold and heat equilibrium states of Yfh1 have a subtle but clear difference in size distribution and compactness.

\section{The two denatured states are differently hydrated}

We then considered whether the two unfolded states could differ by the extent of hydration according to the principles suggested to cause cold rather than heat denaturation. ${ }^{1}$ To address this question, we compared the chemical shifts of the amide protons, which are of course the nuclei most affected by solvation. Differences between the two states become completely evident through this comparison (Fig. 4a). The amide secondary chemical shifts as compared to the $25{ }^{\circ} \mathrm{C}$ random-coil values are consistently different and of opposite sign: the values measured at $-1{ }^{\circ} \mathrm{C}$ are mostly negative, implying deshielding of the protons, whereas those at $50{ }^{\circ} \mathrm{C}$ are mostly positive, indicating shielding effects. These results can only be explained by assuming a different degree of hydrogen bonding of the amides with water.

To convince ourselves that the differences are not due to a trivial chemical shift referencing problem, we compared the HNCBCA spectra at the two temperatures (Fig. 4b). These spectra report the correlation between $\mathrm{HN}, \mathrm{CA}$ and $\mathrm{CB}$ groups so that each strip, cut at a specific $\mathrm{HN}$ frequency (indicated by the number on the $x$-axis of each strip), contains the resonances of the $\mathrm{CA}$ and $\mathrm{CB}$ carbons indicated along the ${ }^{13} \mathrm{C} y$-axis. There is no doubt that the frequencies of the corresponding $\mathrm{HN}$ groups vary widely, while the CA resonances are essentially the same at the two temperatures.

Closer examination of the chemical shifts in terms of different amino acid characteristics also suggests a role of the solvent in promoting unfolding at low temperature. The most interesting comparison is that between hydrophilic and hydrophobic residues. Hydrophobic residues (Fig. S3 of SI) have, on average, larger absolute (downfield) shifts at $-1{ }^{\circ} \mathrm{C}$ with respect to the (highfield) shifts observed at $50{ }^{\circ} \mathrm{C}$. Hydrophilic residues (Fig. S3B of SI) have similar chemical shift changes, albeit still of opposite sign, at $-1{ }^{\circ} \mathrm{C}$ and at $50^{\circ} \mathrm{C}$.

These data show that, despite the global structural similarity between the unfolded states at high and low temperatures, the two states have marked differences in the hydration of amide groups and point to an increased degree of hydration at low temperature.

\section{Comparison of the temperature coefficients of the folded and unfolded species}

We then wondered how much what we are observing would hold for intrinsically unfolded and/or for folded proteins. To assess this, we compared the temperature coefficients of the Yfh1 amide proteins. Plots of the chemical shift values as a function of temperature reveal the presence of two distinct temperature dependences for the folded and unfolded species (Fig. 5a). Interestingly, the temperature coefficients of folded Yfh1 are both positive and negative, probably reflecting a different degree of protection and engagement in intramolecular or intermolecular hydrogen bonding according to different degrees of exposure of the protons in the structured protein. The coefficients of the unfolded species are instead consistently negative, indicating that increase in the temperature leads to a weakening of the interactions with water (Fig. 5b and Fig. S4 of SI). While all negative, the coefficients are not all the same, suggesting different degrees of exposure.

Finally, we used the average temperature coefficients to calculate back the $\Delta \delta\left(\delta_{\mathrm{T}}-\delta_{25}{ }^{\circ} \mathrm{C}\right)$ dependence as a function of temperature for folded 
(a)

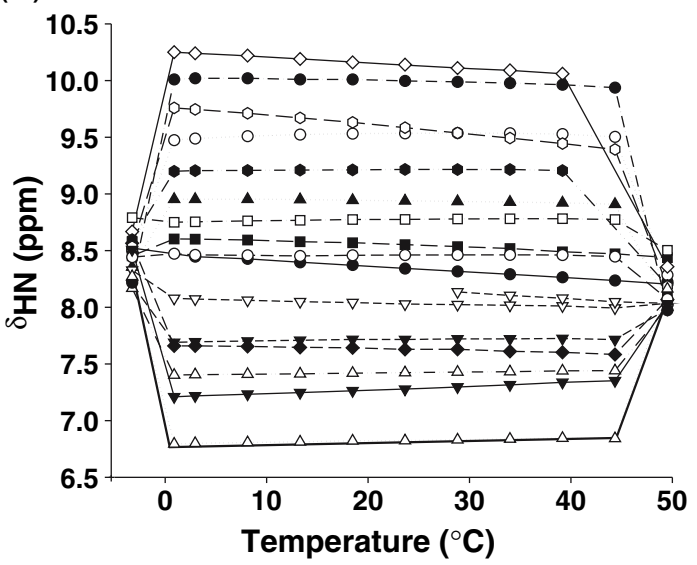

(c)

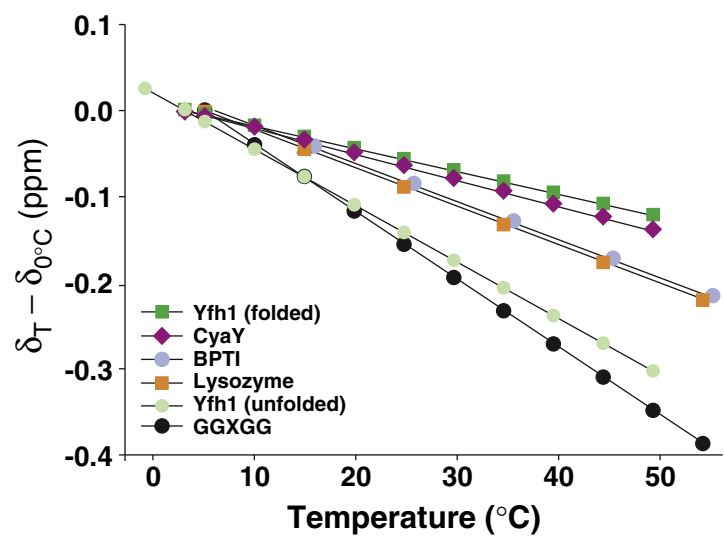

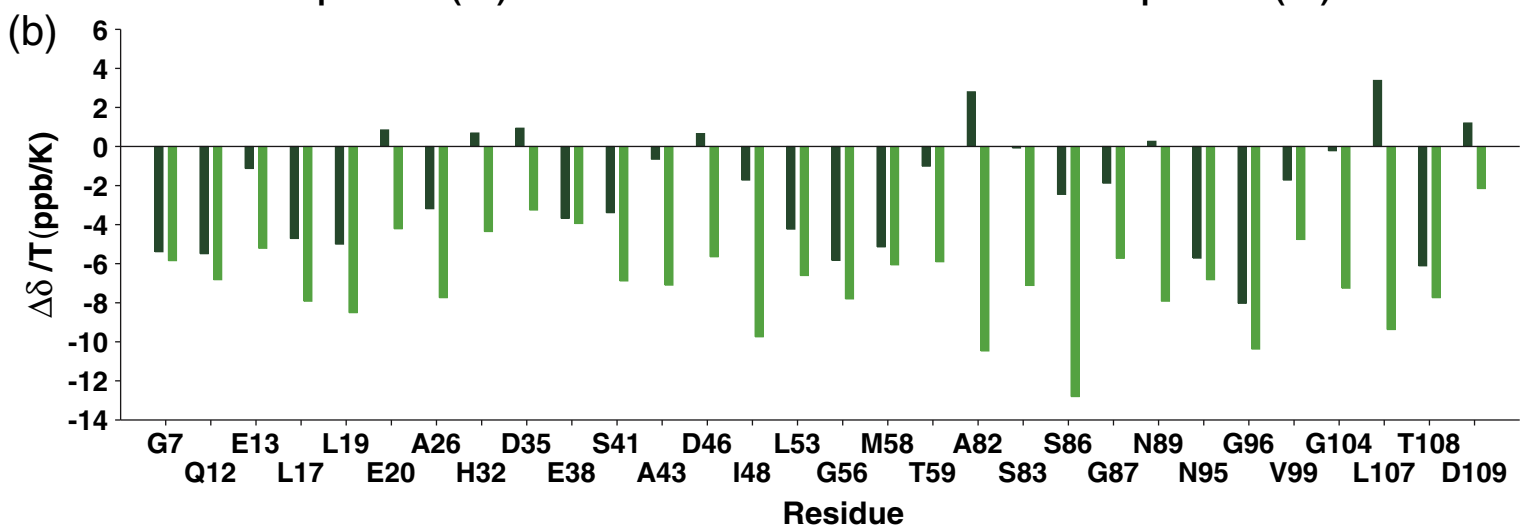

Fig. 5. Temperature coefficient analysis. (a)Plots of the amide proton chemical shifts $\left(\mathrm{d}_{\mathrm{HN}}\right)$ as a function of temperature for representative resonances chosen among the best spread signals (see Fig. S4 of SI). Symbols referring to the same protons are connected only for clarity. (b) Comparison of the temperature coefficients of representative resonances obtained for the folded (dark green) and unfolded (light green) states of Yfh1. (c) Comparison of the chemical shift variation $\left(\delta_{\mathrm{T}}-\delta_{0}{ }^{\circ} \mathrm{C}\right)$ as calculated for the 20 model GGXGG peptides in water ( $\mathrm{pH} 5.5$, black symbol), for the unfolded resonances of Yfh1 ( $\mathrm{pH} 7$, light green), BPTI ( $\mathrm{pH} 4.6$ blue gray), lysozyme ( $\mathrm{pH}$ 5, yellow), CyaY ( $\mathrm{pH} 7$, red) and the folded state of Yfh1 (dark green). The values at each temperature were calculated using published temperature coefficients ${ }^{21}$ (for BPTI, lysozyme and GGXGG) or our experimental data (for Yfh1 and CyaY).

and unfolded Yfh1 and compared the results with plots calculated, using temperature coefficients taken from the literature, for BPTI and lysozyme, ${ }^{21}$ two globular and stably folded proteins, and for model GGXGG peptides that should have features close to random coils. ${ }^{14}$ We also compared the plot calculated for CyaY, the bacterial frataxin orthologue, a whole compact globular protein with the same secondary structure as Yfh1 (except for the Nterminal tail that is absent in CyaY) but very different stability. We observe a clear separation between the slopes of folded proteins and randomcoil states with the latter being steeper (Fig. 5c). This is consistent with the reasonable assumption that, in the unfolded states, the amide protons are on average all more exposed.

These results confirm that the denatured states have features different from those of the folded states and close to those of intrinsically unfolded proteins.

\section{MD simulations confirm increased polar solvation at low temperature}

The picture arising from the NMR data presented here prompted for a model-based analysis of the differences in hydration of Yfh1 over the temperature range of cold and heat denaturation. To this end, two MD trajectories in water were computed at each of three temperatures, $0{ }^{\circ} \mathrm{C}, 25^{\circ} \mathrm{C}$ and $50{ }^{\circ} \mathrm{C}$, with lengths between $450 \mathrm{~ns}$ and $800 \mathrm{~ns}$. The timescale of the simulations is of course insufficient for observing full unfolding by classical MD (Fig. S5 of SI) but can allow us to detect differences in protein-solvent interaction profiles at the initial stages of the perturbation, that is, early in the unfolding process, which could be indicative of a different role of solvation in Yfh1 at the two temperatures. To extract this information from the simulations, we measured the following quantities for hydrogen bonds between protein and water 


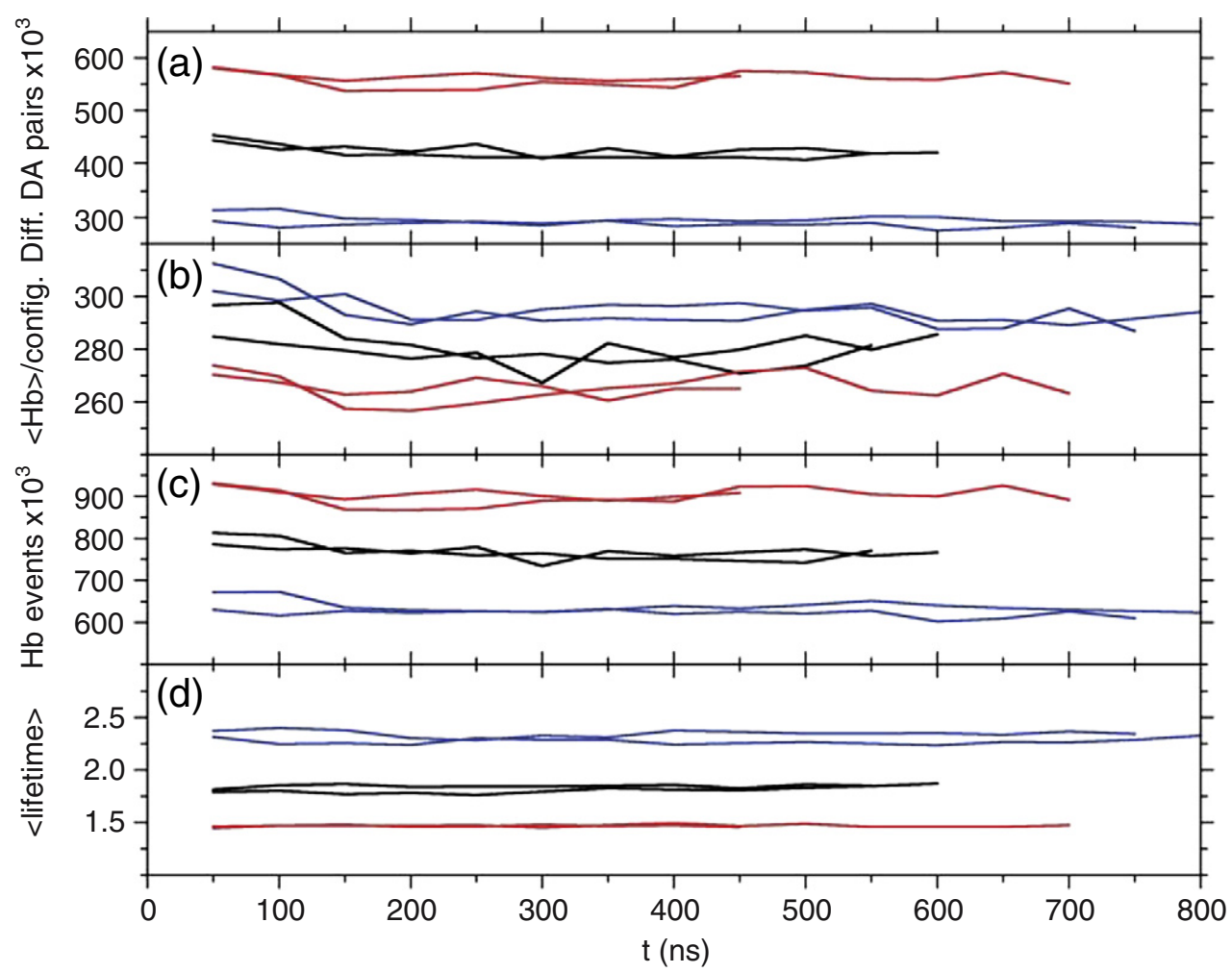

Fig. 6. Hydrogen bonding of Yfh1 and the water at $0{ }^{\circ} \mathrm{C}$ (blue), $25 \mathrm{C}$ (blue), $25^{\circ} \mathrm{C}$ (black) and $50{ }^{\circ} \mathrm{C}$ (red) as calculated from two independent MD trajectories. (a) Number of different donor-acceptor pairs. (b) Average number of H-bonds per configuration. (c) Number of H-bonding events. (d) Average number of consecutive configurations per H-bonding event. The data were calculated for time intervals of 50 ns, with a resolution of one system configuration per 10 ps (i.e., 5000 configurations analyzed per interval). The data point at time $50 \mathrm{~ns}$ thus corresponds to the interval 0-50 ns, and so on. Since the time resolution of the analysis is $10 \mathrm{ps}$, there is not a precise correspondence between the values in (d) and an average lifetime in picoseconds. For example, if an H-bond is observed in just two consecutive configurations, then 10 ps $<$ H-bond lifetime $<30$ ps.

(Fig. 6): the number of different donor-acceptor pairs, the average number of hydrogen bonds per system configuration, the number of hydrogenbonding events and the average number of consecutive configurations per hydrogen-bonding event. The results show a consistent increase in the number of protein-water hydrogen bonds with decreasing temperature, in line with the $\mathrm{NH}$ chemical shift data and with previous thermodynamic analysis of cold denaturation. ${ }^{1}$ The hydrogen-bond exchange rate decreases with temperature (Fig. 6), thus resulting in a lower number of donor-acceptor pairs and hydrogen-bonding events per unit of time and a larger lifetime of the hydrogen bonds. This suggests an increase in both water order around the protein and interfacial protein-water correlation at low temperature. It is worth noting that these results reflect basic hydration properties in the studied temperature range, largely independent of structure.

The simulations fully support a different role of the solvent at low and high temperatures and suggest that cold denaturation is favored by surface water molecules readily available for the crucial interaction with hydrophobic core apolar side chains.

\section{Discussion}

The phenomenon of cold denaturation is increasingly attracting the interest of the scientific community because of its undoubted importance for understanding the forces that determine protein folding. A particularly interesting question is to understand how cold and heat denatured states compare. Although accepted to be thermodynamically equivalent, no detailed characterization from the structural and kinetic point of view is available probably because of the difficulties of studying cold denaturation at accessible temperatures.

Here, we have provided the first direct comparison of the cold and heat denatured states of Yfh1, a natural yeast protein that, under low ionic strength and physiologic conditions, undergoes cold denaturation around $0{ }^{\circ} \mathrm{C}$. An important point that makes our study different from previous ones is 
that, thanks to the unusual properties of Yfh1, we can study the protein without the addition of destabilizing media, such as urea or guanidinium hydrochloride. This is not a trivial interference since addition of chaotropic agents, alcohols or nonphysiologic pHs may change drastically the medium composition and/or the natural protein environment, thus altering the role of solvent on protein stability.

We undertook a detailed site-directed analysis by NMR, a technique that can provide an accurate description of the species present in solution, and complemented the information by CD and SAXS experiments. Our data consistently indicate that although sharing most features of intrinsically unfolded proteins, the ensembles at $-1{ }^{\circ} \mathrm{C}$ and at $50{ }^{\circ} \mathrm{C}$ present important differences. The two transitions occur with a small but genuine asymmetry. To make sure that our studies addressed the very heart of the question of how much the temperature unfolded states differ, we first made sure that the ensembles studied would not contain a mixture of folded and unfolded populations as was the case described in a recent cold denaturation study of a mutant of the L9 protein. $^{22}$ Surprisingly, the temperatures we selected to have reasonably pure unfolded states are not symmetrical along the $\Delta G$ curve, suggesting different kinetics of interconversion.

The residual secondary structure contents also have subtle but clear differences at the two temperatures. Even clearer is the difference observed in parameters that reflect the level of hydration of the molecule: a detailed comparison of the chemical shifts and temperature coefficients tells us that the amide protons, which are expected to be directly influenced by exchange with the solvent, experience a different degree of solvation at high and low temperatures. A different interplay with water is also supported by the different size distribution profiles yielded by the SAXS analysis and is in line with pulsed-field gradient NMR diffusion data. ${ }^{23}$

Taken together, these observations all indicate that the kinetics of interconversion between the folded and unfolded states are different at low and high temperatures because of the interaction with water. It seems fair to hypothesize that tight binding of water molecules to the amide groups can hinder the back conversion from random conformation to local transient secondary elements and determine a different conformational averaging. At high temperature, the amide hydrogens are more weakly solvated, hence allowing a much easier interconversion between partially folded and random states. This interpretation is in line with the importance of dehydration for protein folding suggested by a recent Fourier transform infrared spectrometry study of carbonmonoxymyoglobin and apo myoglobin. ${ }^{24}$
Our experimental observations were further rationalized by MD simulations that provide a detailed description of the microscopic states that precede the catastrophic transitions at which the folded state becomes unstable. We assumed that the strong hydration observed experimentally at the lowest attained temperatures is the result of a progressively increasing process that starts at room temperature and increases with lowering the temperature. By following protein-water hydrogen bonding as a function of time and temperature, we observed an increased solvation of polar groups and a decrease in the hydrogen-bond exchange rate at low temperature, in line with the NMR data. Similar conclusions were reached by other recent MD studies (see, for instance, Refs. 25 and 26). Particularly noteworthy is the exhaustive study of apomyoglobin carried out at temperatures comparable to those used here, which shows an increase in the number of solvent contacts with the protein at low temperature, especially around nonpolar atoms, with concomitant decrease in fluctuations. ${ }^{27}$ The more favorable water-protein interaction was proposed to destabilize the overall protein structure and lead to cold denaturation.

Our observations bear important consequences, in the light of the current interpretation of the cold denaturation phenomenon. ${ }^{1}$ They provide the first direct evidence in support of the present view of the molecular basis of cold denaturation. The process of cold denaturation is characterized by both entropic and enthalpic decreases, that is, just like the process of folding at physiological temperature. This counterintuitive behavior has been explained by the increase in protein hydration with decreasing temperature. ${ }^{1}$ The surprising decrease in entropy upon unfolding is meant to be due to the increase in first-shell water surface in the system-shell water being more ordered than bulk water-associated with the increase in the protein solvent-accessible surface. The ordering of water around the unfolded protein introduces further contributions to the entropy decrease. At low temperature, the water and protein-water correlation terms would compensate for the expected increase in entropy of the protein chain upon unfolding. However, the entropic resultant acting against unfolding would be counteracted by the favorable enthalpic contribution of protein hydration (notably including nonpolar hydration) that is enhanced at low temperature. ${ }^{28}$

In conclusion, this is the first detailed experimental comparison of the structure and properties of the cold and heat denatured states. The picture that comes from our data confirms a leading role of water in the unfolding process. Further studies will be needed to assess the significance of the small difference in residual structure observed in the two ensembles at low and high temperatures. 


\section{Experimental Procedures}

\section{Sample preparation}

Recombinant Saccharomyces cerevisiae Yfh1 was produced as previously described. ${ }^{10,29}$ In short, the protein was expressed in Escherichia coli BL21-(DE3) cells grown in Luria broth at $37^{\circ} \mathrm{C}$, induced in $1 \mathrm{mM}$ IPTG for $6 \mathrm{~h}$, lysed with a French press and sonicated. The soluble overexpressed protein was purified by two ammonium sulfate precipitation steps with a $40 \%$ cut to precipitate contaminating proteins and a $65 \%$ cut to precipitate Yfh1. After dialysis, the protein was subjected to anion-exchange chromatography using a Pharmacia Q-Sepharose column with a gradient to $1 \mathrm{M} \mathrm{NaCl}$, followed by a Pharmacia phenyl-Sepharose column with a decreasing $1 \mathrm{M}$ ammonium sulfate gradient. An ethylenediaminetetraaceticacid-containing protease inhibitor cocktail tablet (Roche) was added before cell lysis and each purification step. Ethylenediaminetetraacetic acid and salts were removed by dialysis prior to concentration of the protein. ${ }^{15} \mathrm{~N}$ labeled and ${ }^{15} \mathrm{~N},{ }^{13} \mathrm{C}$-double-labeled samples were produced by growing the bacteria in minimal medium using labeled ammonium sulfate and glucose as the sole source of nitrogen and carbon.

\section{Far-UV CD measurements}

Far-UV CD spectra were recorded on a Jasco J-715 spectropolarimeter fitted with a cell holder thermostatted by PTC 348-WI Peltier unit. Measurements were carried out on the same samples used for NMR studies after dilution to a protein concentration of $10 \mu \mathrm{M}$. The samples were all in a $20 \mathrm{mM}$ Hepes buffer at pH 7.0 and $2 \mathrm{mM}$ DTT and carefully desalted by dialysis since even small differences in desalting and/or buffer composition are known to influence the Yfh1 stability. ${ }^{4,30}$ Fused silica cuvettes of $1 \mathrm{~mm}$ path length (Hellma) were used. CD spectra were typically recorded with $0.2 \mathrm{~nm}$ resolution and base-line corrected by subtraction of the appropriate buffer spectrum. Thermal unfolding curves were obtained by monitoring the ellipticity at $222 \mathrm{~nm}$ using 2-mm-pathlength cells and a heating rate of $1{ }^{\circ} \mathrm{C} / \mathrm{min}$. Each measurement was repeated at least twice on independent protein preparations to ensure reproducibility of the results.

\section{NMR spectroscopy}

${ }^{15} \mathrm{~N}$ - and ${ }^{15} \mathrm{~N},{ }^{13} \mathrm{C}$-labeled Yhf1 samples used for NMR studies (ca $0.5 \mathrm{mM}$ ) were dialyzed to $20 \mathrm{mM}$ Hepes at $\mathrm{pH} 7.0$ and $2 \mathrm{mM}$ DTT, containing $10 \%(\mathrm{v} / \mathrm{v}) \mathrm{D}_{2} \mathrm{O}$. All experiments were carried out on a Bruker Avance spectrometer operating at $16.4 \mathrm{~T}$ and equipped with an inverse triple-resonance single-axis gradient cryoprobe. Proton chemical shifts were referenced to the water signal fixed at 5.03 and $4.53 \mathrm{ppm}$ for the cold and heat temperature spectra, respectively. ${ }^{13} \mathrm{C}$ and ${ }^{13} \mathrm{~N}$ chemical shifts were referenced indirectly using the ${ }^{1} \mathrm{H}, \mathrm{X}$ frequency ratios of the zero point. ${ }^{31}$ All spectra were processed with NMRPipe/NMRDraw ${ }^{32}$ and analyzed by XEASY/CARA software. ${ }^{33}$
Sequence-specific spectral assignment at $-1{ }^{\circ} \mathrm{C}$ using standard strategies was previously described. ${ }^{8}$ It allowed full and partial assignment of $81 \%$ and $7 \%$ of the residues, respectively, leaving $12 \%$ of the residues unassigned. Assignment at $50{ }^{\circ} \mathrm{C}$ was achieved following the same strategy. Complete assignment at $50^{\circ} \mathrm{C}$ could be achieved for $91 \%$ of the residues. Partial assignment (HA, HB, CA and $\mathrm{CB}$ resonances) was achieved for $7 \%$ of the remaining residues (E2, K21, E39, H55, I70, K72, K77, R90, R102), leaving 2\% (M1 and P74) unassigned. The assignments at both temperatures are deposited in the BioMagResBank $\dagger$ under accession numbers 17068 and 17641, respectively.

Temperature coefficients were calculated by fitting data collected at different temperatures assuming a linear dependence. SSP scores ${ }^{17}$ were calculated using a server online + .

\section{SAXS scattering measurements and data processing}

Synchrotron radiation X-ray scattering data were collected on the European Molecular Biology Laboratory X33 beamline at the DORIS III storage ring (DESY, Hamburg). ${ }^{34}$ Previously desalted solutions of Yfh1 in $20 \mathrm{mM}$ Hepes (pH 7) at $5.0 \mathrm{mg} / \mathrm{ml}$ were measured at 0,10 , 20, 40 and $50{ }^{\circ} \mathrm{C}$ on different aliquots from the same protein batch. Before the measurements, $2 \mathrm{mM}$ DTT was added to the samples to reduce radiation damage. All measurements were performed using the automated SAXS sample change ${ }^{35}$ where the samples are kept in a temperature-controlled sample tray and injected into the independently temperature controlled measuring cell. A 1-M PILATUS detector (DECTRIS, Switzerland) was used at a sample-detector distance of $2.7 \mathrm{~m}$ and a wavelength $\lambda$ of $0.15 \mathrm{~nm}$, covering the momentum transfer range of $0.1<s<5.0 \mathrm{~nm}^{-1}[s=4 \pi \sin (\theta / \lambda)$, where $2 \theta$ is the scattering angle]. The data were processed in the ATSAS program package. ${ }^{36}$ No measurable radiation damage was detected by comparison of four successive time frames with 30-s exposures. The data were averaged after normalization to the intensity of the incident beam. The scattering of the buffer was subtracted and the difference data were scaled and merged and initial analysis was performed using PRIMUS. ${ }^{37}$ The radius of gyration $R_{\mathrm{g}}$ of the solute protein molecule was evaluated using the Guinier approximation at small angles $\left(s<1.3 / R_{\mathrm{g}}\right){ }^{38}$ The $\mathrm{EOM}^{20}$ was used to assess the equilibrium of folded and unfolded states of Yfh1 under cold and heat denaturation conditions.

\section{MD simulations}

MD simulations of yeast frataxin were carried out at $0{ }^{\circ} \mathrm{C}, 25{ }^{\circ} \mathrm{C}$ and $50{ }^{\circ} \mathrm{C}$. The simulations were performed with the software package GROMACS 3.3.1, ${ }^{39,40}$ using the GROMOS $45 \mathrm{a} 3$ force field ${ }^{41,42}$ in combination with the SPC water model. ${ }^{43}$ Simulations were run under periodic boundary conditions at the NPT thermodynamic ensemble. Temperature and pressure (1 atm) were kept constant by a weak-coupling method, ${ }^{44}$ with relaxation times of 0.1

$\dagger$ http:/ / www.bmrb.wisc.edu

thttp:/ / pound.med.utoronto.ca/software.html 
and 0.5 ps, respectively, and independent coupling of solute and solvent to the temperature bath. The equations of motion were integrated with the leap-frog algorithm using a time step of $2 \mathrm{fs}$. Protein bond lengths were constrained with LINCS, ${ }^{45}$ while the geometry of the water model was constrained with SETTLE. ${ }^{46}$ Nonbonded interactions were calculated using a twin-range scheme plus a reaction field long-range correction, ${ }^{47}$ with cutoff radii of $0.8 \mathrm{~nm}$ and $1.4 \mathrm{~nm}$, a pair-list update frequency of 5 steps and a dielectric constant outside the 1. 4-nm cutoff sphere of 78. The initial system included 1 protein copy (Protein Data Bank code 2GA5), 11,286 water molecules and 15 randomly placed $\mathrm{Na}^{+}$neutralizing the total protein charge, in a rhombic dodecahedron with walls at a minimum distance of $1.1 \mathrm{~nm}$ from any protein atom. The system was energy minimized and progressively brought to the reference temperature in 100 ps. Two independent runs of varying length (between $450 \mathrm{~ns}$ and $800 \mathrm{~ns}$ ) were carried out for each temperature. Trajectory coordinates and energies were stored every $10 \mathrm{ps}$. Secondary structure analysis was performed with $\mathrm{DSSP}^{48}$ and characterization of hydrogen bonding with the analysis tools available in the Gromacs package and ad hoc scripts.

\section{Acknowledgements}

We gladly acknowledge financial support from an International JOINT PROJECT of the Royal Society (NIMR: U.1175.03.002.00001.04). D.U. was recipient of a postdoctoral fellowship from Autonomous University of Barcelona. P.V.K and D.I.S. acknowledge financial support from FP7 e-Infrastructure project WeNMR (contract number 261572).

\section{Supplementary Data}

Supplementary data to this article can be found online at doi:10.1016/j.jmb.2012.02.002

\section{References}

1. Privalov, P. L. (1990). Cold denaturation of proteins. Crit. Rev. Biochem. Mol. Biol. 25, 281-305.

2. Whitten, S. T., Kurtz, A. J., Pometun, M. S., Wand, A. J. \& Hilser, V. J. (2006). Revealing the nature of the native state ensemble through cold denaturation. Biochemistry, 45, 10163-10174.

3. Szyperski, T. \& Mills, J. L. (2011). NMR-based structural biology of proteins in supercooled water. J. Struct. Funct. Genomics, 12, 1-7.

4. Pastore, A., Martin, S., Politou, A., Kondapalli, K. C., Stemmler, T. \& Temussi, P. A. (2007). Unbiased cold denaturation: low- and high-temperature unfolding of yeast frataxin under physiological conditions. J. Am. Chem. Soc. 129, 5374-5375.

5. Pandolfo, M. \& Pastore, A. (2009). The pathogenesis of Friedreich ataxia and the structure and function of frataxin. J. Neurol. 256, 9-17.
6. Adinolfi, S., Rizzo, F., Masino, L., Nair, M., Martin, S., Pastore, A. \& Temussi, P. A. (2004). The factors governing the thermal stability of frataxin orthologues: how to increase a protein's stability. Biochemistry, 43, 6511-6518.

7. Martin, S. R., Esposito, V., De Los Rios, P., Pastore, A. \& Temussi, P. A. (2008). Cold denaturation of yeast frataxin offers the clue to understand the effect of alcohols on protein stability. J. Am. Chem. Soc. 130, 9963-9970.

8. Adrover, M., Esposito, V., Martorell, G., Pastore, A. \& Temussi, P. A. (2010). Understanding cold denaturation: the case study of Yfh1. J. Am. Chem. Soc. 132, 16240-16246.

9. Karlberg, T., Schagerlöf, U., Gakh, O., Park, S., Ryde, U., Lindahl, M. et al. (2006). The structures of frataxin oligomers reveal the mechanism for the delivery and detoxification of iron. Structure, 14, 1535-1546.

10. He, Y., Alam, S. L., Proteasa, S. V., Zhang, Y., Lesuisse, E., Dancis, A. \& Stemmler, T. L. (2004). Yeast frataxin solution structure, iron binding, and ferrochelatase interaction. Biochemistry, 43, 16254-16262.

11. Gordon, D. M., Kogan, M., Knight, S. A., Dancis, A. \& Pain, D. (2001). Distinct roles for two N-terminal cleaved domains in mitochondrial import of the yeast frataxin homolog, Yfh1p. Hum. Mol. Genet. 10, 259-269.

12. Uversky, V. N. (2011). Intrinsically disordered proteins may escape unwanted interactions via functional misfolding. Biochim. Biophys. Acta, 1814, 693-712.

13. Wong, K. B., Freund, S. M. \& Fersht, A. R. (1996). Cold denaturation of barstar: ${ }^{1} \mathrm{H},{ }^{15} \mathrm{~N}$ and ${ }^{13} \mathrm{C}$ NMR assignment and characterisation of residual structure. J. Mol. Biol. 259, 805-818.

14. Kjaergaard, M., Brander, S. \& Poulsen, F. M. (2011). Random coil chemical shift for intrinsically disordered proteins: effects of temperature and $\mathrm{pH}$. J. Biomol. NMR, 49, 139-149.

15. Wishart, D. S. \& Sykes, B. D. (1994). Chemical shifts as a tool for structure determination. Methods Enzymol. 239, 363-392.

16. Pastore, A. \& Saudek, V. (1990). The relationship between chemical shift and secondary structure in proteins. J. Magn. Reson. 90, 165-176.

17. Marsh, J. A., Singh, V. K., Jia, Z. \& Forman-Kay, J. D. (2006). Sensitivity of secondary structure propensities to sequence differences between alpha- and gammasynuclein: implications for fibrillation. Protein Sci. 15, 2795-2804.

18. Merutka, G., Dyson, H. J. \& Wright, P. E. (1995). 'Random coil' ${ }^{1} \mathrm{H}$ chemical shifts obtained as a function of temperature and trifluoroethanol concentration for the peptide series GGXGG. J. Biomol. NMR, 5, 14-24.

19. Shkumatov, A. V., Chinnathambi, S., Mandelkow, E. \& Svergun, D. I. (2011). Structural memory of natively unfolded tau protein detected by small-angle X-ray scattering. Proteins, 79, 2122-2131.

20. Bernadó, P., Mylonas, E., Petoukhov, M. V., Blackledge, M. \& Svergun, D. I. (2007). Structural characterization of flexible proteins using small-angle X-ray scattering. J. Am. Chem. Soc. 129, 5656-5664.

21. Baxter, N. J. \& Williamson, M. P. (1997). Temperature dependence of ${ }^{1} \mathrm{H}$ chemical shifts in proteins. J. Biomol. NMR, 9, 359-369. 
22. Shan, B., McClendon, S., Rospigliosi, C., Eliezer, D. \& Raleigh, D. P. (2010). The cold denatured state of the C-terminal domain of protein 19 is compact and contains both native and non-native structure. J. Am. Chem. Soc. 132, 4669-4677.

23. Li, Y., Shan, B. \& Rayleigh, D. P. (2007). The cold denatured state is compact but expands at low temperatures: hydrodynamic properties of the cold denatured state of the C-terminal domain of L9. J. Mol. Biol. 368, 256-262.

24. Guo, L., Park, J., Lee, T., Chowdhury, P., Lim, M. \& Gai, F. (2009). Probing the role of hydration in the unfolding transitions of carbonmonoxy myoglobin and apomyoglobin. J. Phys. Chem. B, 113, 6158-6163.

25. Mallamace, F., Corsaro, C., Mallamace, D., Baglioni, P., H. Stanley, H. E. \& Chen, S. H. (2011). A possible role of water in the protein folding process.J. Phys. Chem. B, Oct 21 Epub ahead of print.

26. Segupta, N., Jaud, S. \& Tobias, D. J. (2008). Hydration dynamics in a partially denatured ensemble of the globular protein human alpha-lactalbumin investigated with molecular dynamics simulation. Biophys. J. 95, 5257-5267.

27. Lopez, C. F., Darst, R. K. \& Rossky, P. J. (2008). Mechanistic elements of protein cold denaturation. J. Phys. Chem. B, 112, 5961-5967.

28. Privalov, P. L. \& Gill, S. J. (1988). Stability of protein structure and hydrophobic interaction. Adv. Protein Chem. 39, 191-234.

29. Adinolfi, S., Trifuoggi, M., Politou, A. S., Martin, S. \& Pastore, A. (2002). A structural approach to understanding the iron-binding properties of phylogenetically different frataxins. Hum. Mol. Genet. 11, 1865-1877.

30. Nair, M., Adinolfi, S., Pastore, C., Kelly, G., Temussi, P. \& Pastore, A. (2004). Solution structure of the bacterial frataxin ortholog, CyaY: mapping the iron binding sites. Structure, 12, 2037-2048.

31. Wishart, D. S., Bigam, C. G., Yao, J., Abildgaard, F., Dyson, H. J., Oldfield, E. et al. (1995). ${ }^{1} \mathrm{H},{ }^{13} \mathrm{C}$ and ${ }^{15} \mathrm{~N}$ chemical shift referencing in biomolecular NMR J. Biomol. NMR, 2, 135-140.

32. Delaglio, F., Grzesiek, S., Vuister, G. W., Zhu, G., Pfeifer, J. \& Bax, A. (1995). NMRPipe: a multidimensional spectral processing system based on UNIX pipes. J. Biomol. NMR, 6, 277-293.

33. Bartels, C., Xia, T. H., Billeter, M., Güntert, P. \& Wüthrich, K. (1995). The program XEASY for computer-supported NMR spectral analysis of biological macromolecules. J. Biomol. NMR, 6, 1-10.

34. Roessle, M. W., Klaering, R., Ristau, U., Robrahn, B., Jahn, D., Gehrmann, T. et al. (2007). Upgrade of the small angle X-ray scattering beamline X33 at the European Molecular Biology Laboratory, Hamburg. J. Appl. Crystallogr. 40, s190-s194.
35. Round, A. R., Franke, D., Moritz, S., Huchler, R., Fritsche, M., Malthan, D. et al. (2008). Automated sample-changing robot for solution scattering experiments at the EMBL Hamburg SAXS station X33. J. Appl. Crystallogr. 41, 913-917.

36. Konarev, P. V., Petoukhov, M. V., Volkov, V. V. \& Svergun, D. I. (2006). ATSAS 2.1, a program package for small-angle scattering data analysis. J. Appl. Crystallogr. 39, 277-286.

37. Konarev, P. V., Volkov, V. V., Sokolova, A. V., Koch, M. H. J. \& Svergun, D. I. (2003). PRIMUS: a Windows PC-based system for small-angle scattering data analysis. J. Appl. Crystallogr. 36, 1277-1282.

38. Guinier, A. (1939). La diffraction des rayous X aux tres petits angles: application a l'etude de phenomenes ultramicroscopiques. Ann. Phys. 12, 161-237.

39. Berendsen, H. J. C., van der Spoel, D. \& van Drunen, R. (1995). GROMACS: A message-passing parallel molecular dynamics implementation. Comput. Phys. Commun. 91, 43-56.

40. Lindahl, E., Hess, B. \& van der Spoel, D. (2001). Gromacs 3.0: a package for molecular simulation and trajectory analysis. J. Mol. Mod. 7, 306-317.

41. van Gunsteren, W. F., Billeter, S. R., Eising, A. A., Hünenberger, P. H., Krüger, P., Mark, A. E. et al. (1996). Biomolecular Simulation: The GROMOS96 Manual and User Guide vdf Hochschulverlag AG an der ETH Zürich and BIOMOS b.v., Zürich and Groningen.

42. Schuler, L. D., Daura, X. \& van Gunsteren, W. F. (2001). An improved GROMOS96 force field for aliphatic hydrocarbons in the condensed phase. J. Comput. Chem. 22, 1205-1218.

43. Berendsen, H. J. C., Postma, J. P. M., van Gunsteren, W. F. \& Hermans, J. (1981). Interaction models for water in relation to protein hydration. In Intermolecular Forces (Pullman, B., ed.), pp. 331-342, D. Reidel Publishing Company, Dordrecht, The Netherlands.

44. Berendsen, H. J. C., Postma, J. P. M., van Gunsteren, W. F., Di Nola, A. \& Haak, J. R. (1984). Molecular dynamics with coupling to an external bath. J. Chem. Phys. 81, 3684-3690.

45. Hess, B., Bekker, H., Berendsen, H. J. C. \& Fraaije, J. G. E. M. (1997). LINCS: a linear constraint solver for molecular simulations. J. Comp. Chem. 18, 1463-1472.

46. Miyamoto, S. \& Kollman, P. A. (1992). SETTLE: an analytical version of the SHAKE and RATTLE algorithm for rigid water models. J. Comp. Chem. 13, 952-962.

47. Tironi, I. G., Sperb, R., Smith, P. E. \& van Gunsteren, W. F. (1995). A generalized reaction field method for molecular dynamics simulations. J. Chem. Phys. 102, 5451-5459.

48. Kabsch, W. \& Sander, C. (1983). Dictionary of protein secondary structure: pattern recognition of hydrogenbonded and geometrical features. Biopolymers, 22, 2577-2637. 